\title{
Thermo-mechanical analysis of Wire and Arc Additive Layer Manufacturing process on large multi-layer parts
}

\author{
J. Ding*¹, P. Colegrove ${ }^{2}$, J. Mehnen ${ }^{1}$, S. Ganguly ${ }^{2}$, P.M. Sequeira Almeida ${ }^{2}$, \\ F. Wang ${ }^{2}$, and S. Williams ${ }^{2}$
}

Wire and Arc Additive Layer Manufacturing (WAALM) is gaining increasing popularity as the process allows the production of large custom-made metal workpieces with high deposition rates. The high power input of the welding process, causes significant residual stress and distortion of the workpiece. This paper describes the thermo-mechanical behaviour of the multi-layer wall structure made by the WAALM process. A 3D thermoelastic-plastic transient model and a model based on an advanced steady-state thermal analysis are employed in this study. The temperature simulations and distortion predictions are verified by comparing with the experimental results from thermo-couples and laser scanners, while the residual stresses are verified with the neutron diffraction strain scanner ENGIN-X. The stress across the deposited wall is found uniform with very little influence of the preceding layers on the following layers. The stress redistributed after unclamping with a much lower value at the top of the wall than at the interface due to the bending distortion of the sample. The FEM model based on the steady-state thermal model shows a significant advantage on the computational time.

Keywords: Additive Layer Manufacturing, Thermo-mechanical analysis, Lagrangian model, Eulerian model, residual stress, distortion.

\section{Introduction}

Additive Layer Manufacturing has become an important industrial process for the manufacture of custom-made metal workpieces. Innovative Wire and Arc Additive Layer Manufacture (WAALM) solutions have emerged recently to fabricate highly reactive metallic components in an out-of-chamber environment (e.g. Ti-6Al-4V) [1]. Faster processing speeds and high deposition rate capabilities are central attributes to enable the production of large-scale aerospace components [2]. Although the wire-added ALM process has been around for almost a century - the first patent was filed 1920 by Baker [3] - modern welding and automation technologies provide opportunities that were not previously available. In addition, there is currently a demand for sustainable, low cost, environmentally friendly processes with high geometric flexibility. Finally, WAALM can be used to deposit a variety of materials that can be welded, such as steel [4, 5], Ni alloys [6], and Ti alloys [1, 7].

In the WAALM process, 3D metallic components are built by depositing beads of weld metal in a layer by layer fashion. Standard wire based welding processes such as Gas Metal Arc Welding (GMAW) and Gas Tungsten Arc Welding (GTAW) are low cost solutions employed as heat sources, providing high deposition rates by utilising high energy input.

However, the high heat input leads to significant distortion and residual stress. Finite Element (FE) analysis can be used to predict the residual stress and distortion during additive manufacturing processes [8-10], enabling the development of mitigation methods $[11,12]$. Most of the FE analyses utilise transient models with a moving heat source. Element birth technique is used for simulating addition of material, providing accurate predictions. However, the model size is usually limited due to the computational time required for these simulations due to the long processing time to make the real parts.

Therefore to develop models of large-scale WAALM components, which can scale to several meters, the conventional transient FE analysis is not suitable. A new method is needed to reduce the calculation time while keeping the simulation accuracy high. For a long weld with a constant welding speed, the thermal histories approach a steady state, particularly in the middle of the weld. This phenomenon has been utilised in FE models of welding processes which have used a steady state model [13-16, 18].

\footnotetext{
${ }^{1}$ Manufacturing Department, Cranfield University,

Cranfield, MK43 OAL, UK

${ }^{2}$ Welding Engineering Research Centre, Cranfield University,

Cranfield, MK43 0AL, UK

*Corresponding author, email jialuo.ding@cranfield.ac.uk
} 
Unlike the conventional transient method which uses a time increment scheme to model the moving welding torch, the steady state method attaches an Eulerian reference frame to the welding torch and the material "flows" through the mesh (Fig. 1). Therefore the problem can be solved for a single time step saving a large amount of computational time. Moreover, the model using the Eulerian frame does not need to use a high density mesh uniformly along the weld line, saving additional computational time [14]. While the steady state solution of the thermal problem is relatively trivial, application to the mechanical problem is more difficult, but has been demonstrated by several authors [13,14, 16, 17]. An alternative approach, developed by Colegrove et al.[18] cut the Eulerian thermal model into a series of temperature data slices, and then transferred the sliced temperature data onto a 2D elastic-plastic model.
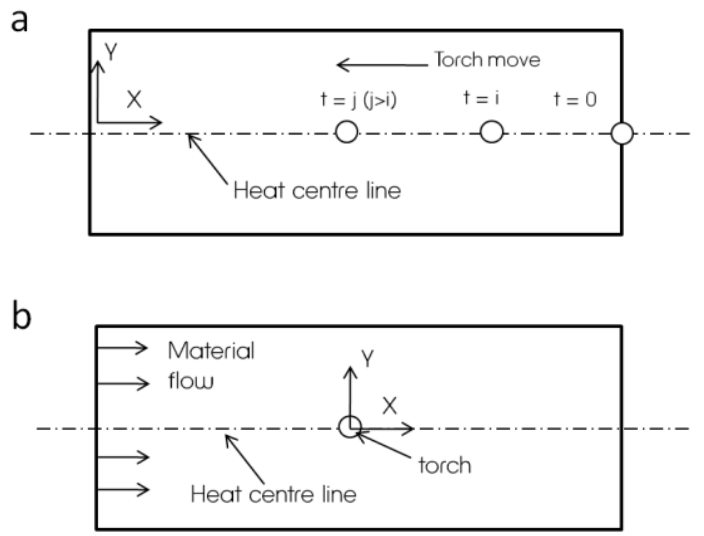

Fig. 1: Model with different reference frames. (a) Lagrangian reference frame (b) Eulerian reference frame.

In this paper, an efficient FE method is developed to analyse the thermo-mechanical performance of the WAALM process on a $500 \mathrm{~mm}$ mild steel multi-layer wall structure. Steady state temperature distributions are calculated using the Eulerian thermal method, which are used as an input to a 3D mechanical model for residual stress and distortion analysis. A comparison was carried out between this efficient FE model and a conventional transient thermo-mechanical model. Both models are compared with experimental measurements of temperature, residual stress and distortion.

\section{Experimental set-up}

Sample walls were produced in mild steel using WAALM. The base plates used in the experiments were rolled structural steel plates (grade S355JR-AR). The chemical composition of the alloy (in wt. \%) is $0.24 \% \mathrm{C}, 1.60 \%$ $\mathrm{Mn}, 0.55 \% \mathrm{Si}, 0.045 \% \mathrm{P}, 0.045 \% \mathrm{~S}, 0.009 \% \mathrm{~N}, 0.003-0.100 \% \mathrm{Nb}$ and Fe balance. The selection of the consumable electrode was based on a strict chemical composition criterion in order to obtain optimum as welded mechanical properties. The chemical composition of the solid wire (in wt. \%) is $0.08 \% \mathrm{C}, 1.50 \% \mathrm{Mn}$, $0.92 \% \mathrm{Si}, 0.16 \% \mathrm{Cu}, \leq 0.040 \% \mathrm{P}, \leq 0.035 \% \mathrm{~S}$ and $\mathrm{Fe}$ balance.

The geometry of the structure is shown in Fig. 2. The walls were deposited along the centreline of the base plate with a width of $5 \mathrm{~mm}$ and a height of $2 \mathrm{~mm}$ for each layer. The heat source is a Cold Metal Transfer (CMT) [23] welding power source, a modified GMAW variant based on a controlled dip transfer mode mechanism, which can provide a high deposition rate capability at a relatively low heat input. The welding wire used in this study is $1.2 \mathrm{~mm}$ and the wire feed speed is $10 \mathrm{~m} \mathrm{~min}^{-1}$. The travel speed of the welding torch is 8.33 $\mathrm{mm} \mathrm{s}^{-1}$. The welding process heat input is $269.5 \mathrm{~J} \mathrm{~mm}^{-1}$, assuming an efficiency of 0.9 . A water cooled aluminium backing plate was utilised in order to cool the sample more rapidly. A waiting time of 400 seconds was used between subsequent layers enabling the sample to cool below $50^{\circ} \mathrm{C}$ before new layers were deposited. Four k-type thermocouples were welded at the positions indicated in Fig. 2.

To verify the mechanical model, residual strain measurements were carried out at the ENGIN-X strain scanner at ISIS, Oxford, UK. Residual stresses data of three directions are taken in the base plate at the position $2 \mathrm{~mm}$ below the top surface and located along the transverse direction of the mid-length section of WAALM samples, which is shown in B-B section of Fig. 2. Residual stresses are also measured along the normal direction of the mid-length section of the deposited wall. 


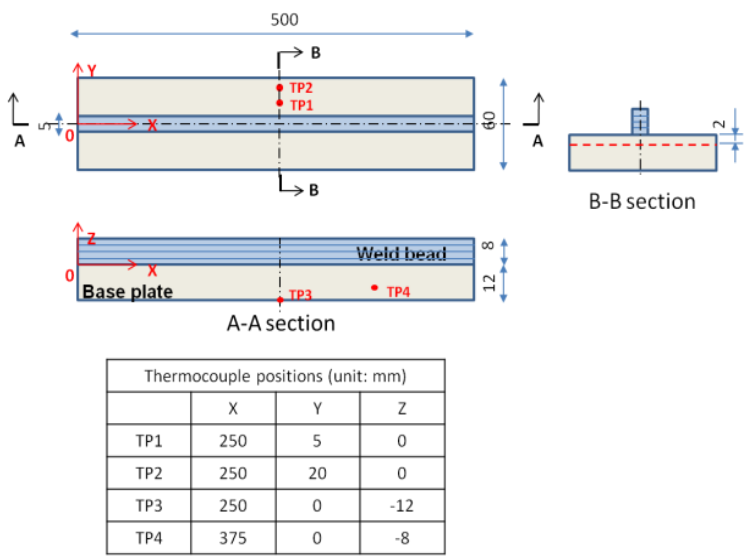

Fig. 2: Geometry of the WAALM sample.

The time-of-flight principle of strain measurement is utilised to determine the lattice strain [24, 25]. The longitudinal strain direction was measured using a gauge volume of $2 \times 2 \times 2 \mathrm{~mm}^{3}$, while the transverse and normal strain directions were measured using a gauge volume of $2 \times 20 \times 2 \mathrm{~mm}^{3}$. The opening of the vertical dimension of the incoming beam during measurement of the transverse and normal strain directions would lead to a faster measurement with higher neutron flux without the loss of any spatial resolution. The data analysis was performed for a time-of-flight spectrum of 20-40 milliseconds which contains the $\{110\},\{200\}$ and $\{211\}$ families of crystallographic planes. The range and families of crystallographic planes analysed ensured an average macroscopic strain determination free from crystal anisotropy. The diffraction spectrum was analysed by Pawley refinement [26] using GSAS software. References for strain calculations were measured in the far field parent in a $3 \mathrm{~mm}$ thick slice removed from the edge of the specimens. A constant stress free value was used for the calculations of the strain in the base plate because the penetration level of the deposited wire would not affect the composition of the measurement location in the base plate. Different reference slice was used for the measurements on the deposited wall.

Strain measured in all the three directions were then combined to analyse the stress assuming the measured directions are the principal strain directions and following Hooke's law as shown in equation 1.

$$
\sigma_{x}=\frac{E}{(1+v)} \varepsilon_{x}+\frac{E v}{(1+v)(1-2 v)}\left(\varepsilon_{x}+\varepsilon_{y}+\varepsilon_{z}\right)
$$

Where $\varepsilon_{x}, \varepsilon_{y}$ and $\varepsilon_{z}$ are the strains in the principal directions; $\mathrm{E}$ is the elasticity of the material; and $v$ is Poisson's ratio of the material.

In addition, distortion was taken from the sample with 4 layer wall using a Romer Omega Arm with R-Scan 3D laser scanning system.

\section{Thermo-mechanical FEM models}

\subsection{FE thermal models}

The FE software package, ABAQUS [19] was used for both the thermal and mechanical models. Sequentially coupled thermo-mechanical simulations were carried out which calculated the temperature distribution first and then used the results for the mechanical analysis. Two model types were developed. The first used a steady state thermal model which used an Eulerian reference frame, and the second used a transient model and Lagrangian reference frame. Only half of the material is modelled due to the symmetry along the central axis which shown as A-A in Fig. 2.

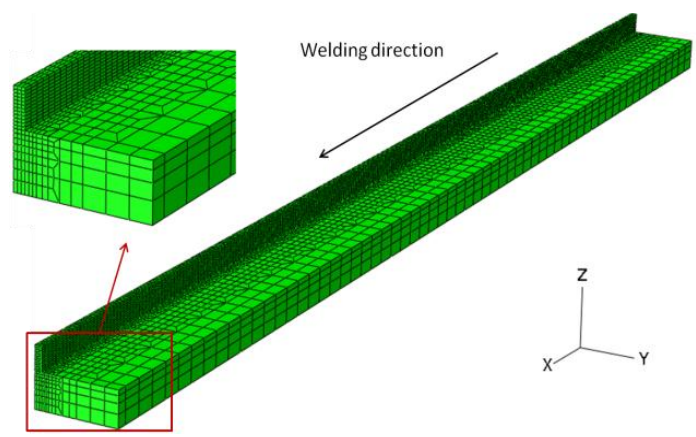

Fig. 3: Mesh of transient thermal model. 
For the Lagrangian thermal model, linear brick elements with 8 nodes (DC3D8) are used for the thermal simulation. To capture the thermal performance around the heat source, dense meshes of size $2 \mathrm{~mm} \times 0.833 \mathrm{~mm}$ $\times 0.667 \mathrm{~mm}$ were used for the bead and the area near welding line. The meshes became coarser in the $\mathrm{y}$ direction and $-\mathrm{z}$ direction away from the welding line (Fig. 3). The filler material was simulated using the "element birth technique" [21]. All the elements of the bead are deactivated at the first step of the analysis, and then the elements are activated sequentially following the heat source.

The moving heat source for the Lagrangian thermal model is generated with the user subroutine DFLUX in the ABAQUS code. The Goldak double ellipsoidal heat source [20] was used to apply the heat to the additive manufacture deposits. The power density of the region in front of the arc centre and the region behind the arc centre is defined separately using:

$$
\begin{aligned}
& q_{f}=\frac{6 \sqrt{3} Q f_{f}}{\pi \sqrt{\pi} a_{f} b c} e^{\left[-3\left(\frac{x^{2}}{a_{f}^{2}}+\frac{y^{2}}{b^{2}}+\frac{z^{2}}{c^{2}}\right)\right]} \\
& q_{r}=\frac{6 \sqrt{3} Q f_{r}}{\pi \sqrt{\pi} a_{r} b c} e^{\left[-3\left(\frac{x^{2}}{a_{r}^{2}}+\frac{y^{2}}{b^{2}}+\frac{z^{2}}{c^{2}}\right)\right]} \\
& f_{f}+f_{r}=2
\end{aligned}
$$

Where $a_{f}$ and $a_{r}$ are the length of the frontal ellipsoid and the rear ellipsoid, respectively; $b$ is the width of the heat source; $c$ is the depth of the heat source; $Q$ is the energy input considering the factor of efficiency; $f_{f}$ and $f_{r}$ are the factors for distributing the power to the front and rear of the hear source.

Detailed parameter settings for the heat source model used in this paper are shown in Table 1.

Table 1: Heat source parameters

\begin{tabular}{|c|c|c|c|c|c|c|}
\hline$a_{f}(\mathrm{~mm})$ & $a_{r}(\mathrm{~mm})$ & $b(\mathrm{~mm})$ & $c(\mathrm{~mm})$ & $Q(\mathrm{~W})$ & $f_{f}$ & $f_{r}$ \\
\hline 2 & 6 & 2.5 & 3 & 2245.83 & 0.6 & 1.4 \\
\hline
\end{tabular}

Fig. 4 shows the mesh used for the steady-state model. Unlike the transient model, the steady-state model does not require a uniform mesh with high density along the weld line. Forced convection/ diffusion brick elements (DCC3D8) were utilised in this model. The smallest elements are located in the heating area with a size of $1.667 \mathrm{~mm} \times 0.833 \mathrm{~mm} \times 0.667 \mathrm{~mm}$, while the largest ones are at the outlet face with the size of 344.656 $\mathrm{mm} \times 5.407 \mathrm{~mm} \times 2.712 \mathrm{~mm}$. A mass flow rate per area, which equals to the welding speed multiply with the material density, is used to represent the torch movement. The heat distribution for the steady state model is also represented with the same Goldak double ellipsoidal heat source.

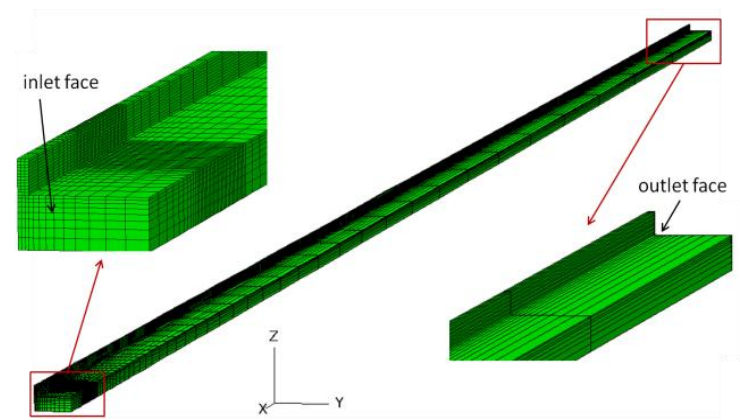

Fig. 4: Mesh of the steady state thermal model.

As the component did not cool down to ambient temperature between deposits it was necessary to include the effect of the residual temperature in the models for the $2^{\text {nd }}, 3^{\text {rd }}$ and $4^{\text {th }}$ layers. This was done by taking the nodal temperatures from the outlet surface for the previous model and applying them to the inlet surface of the model for the next layer. To provide an accurate prediction of the outlet temperature the length of the model is set so that:

$$
\mathrm{L}=\mathrm{V}_{\text {torch }} \times \mathrm{T}_{\text {waiting }}+\mathrm{L}_{\text {actual }}
$$

Where $\mathrm{L}$ is the length of the Eulerian thermal model;

$\mathrm{V}_{\text {torch }}$ is the travel speed of the welding torch; $\mathrm{T}_{\text {waiting }}$ is the waiting time between subsequent layers; and $\mathrm{L}_{\text {actual }}$ is the actual length of the sample.

Using this approach, the effective cooling time between subsequent layers is identical to the experimental condition. 
Both models used thermal material properties from Zhang and Michaleris [16]. An artificially high thermal conductivity is used for temperatures greater than $1500^{\circ} \mathrm{C}$ to capture the convective heat transfer in the weld pool. The radiation coefficient and the convection coefficient are assumed independent of the temperature and are set to 0.2 and $5.7 \mathrm{Wm}^{-2} \mathrm{~K}^{-1}$, respectively. The heat loss through the cooling system under the base plate is modelled with an equivalent convection coefficient. This coefficient was found by running a series of numerical trials, and tuning the value so that the predicted temperature profiles matched the experimental results. A value of $300 \mathrm{Wm}^{-2} \mathrm{~K}^{-1}$ gave the best match with the experiments.

\subsection{FE mechanical models}

The Lagrangian mechanical model uses the same meshing as the Lagrangian thermal model, but the mesh type is changed to integrated 3D 8-node element (C3D8R). The steps for the mechanical simulation are set with the same number and time as the thermal simulation. The nodal temperature results calculated from the thermal analysis are utilised as thermal load input for the mechanical simulation.

A 3D elastic-plastic mechanical model is used to calculate the residual stress from the steady state thermal model data. The length of the mechanical model was $500 \mathrm{~mm}$ which was the actual length of the experimental sample. It was meshed with the same mesh spacing as the steady state model in the $\mathrm{y}$ and $\mathrm{z}$ directions, however the spacing in the $x$ direction was set to a constant value of $2.5 \mathrm{~mm}$.

To use steady state thermal results as the input to the mechanical model, the static nodal temperature data were transferred to thermal histories by dividing the position in the $\mathrm{x}$ direction by the travel speed. Temperature histories were added sequentially using the results from the model with one layer bead to the model with four layer bead. The stress analysis was carried out by applying the transferred thermal history sequentially to the 3D mechanical model. The temperature field from the steady state model is effectively mapped to the mechanical model using a pre-processing programme generated in Matlab.

"Element birth technique" was utilised in both mechanical models for simulating the adding process of the filler materials. Both models used temperature dependant mechanical properties from Zhang and Michaleris [16]. The phase transformations are ignored in this study, as it has an insignificant effect on the welding residual stress for mild steel [22]. To avoid difficulties in convergence, a 'cut-off temperature' of $1000^{\circ} \mathrm{C}$ was used in the material model. The Young's modulus and the yield stress of the material remain at the same value for temperatures above the cut off temperature.

To simulate the clamping system used in the WAALM process, a 'backing plate' was included with rigid material properties. A hard contact is applied between the contact surface of the base plate and the backing plate. This prevents the points on the base plate to moving in $-\mathrm{Z}$ direction but not against the movement in $+\mathrm{Z}$ direction. Clamps in the real WAALM experiment are located on the four corners and the mid length of the base plate. This was modelled by fixing the movements of the nodes at the corresponding positions. The clamping constraints were removed in the stages when the base plate was unclamped.

\subsection{Time comparison}

The comparison of the computational time used for the two models is presented in Table 2. All the simulations were calculated on a grid computing system with four processors. The computational time consumed by the transient thermal model can be greatly reduced by using the steady-state model. The transient thermal model provides much more data than the steady state model as it calculates the incremental temperature histories, which result in more time increments needed for completing the mechanical analysis. The number of time increment for completing one step with transient mechanical model is 32 increments in average, while the mechanical model using the mapped steady state thermal results needs only 20 increments to complete the same step. Thus, the time taken for the mechanical model based on the steady-state model was reduced by around $40 \%$. Hence the total time saving with the new model is around $80 \%$.

Table 2: Computational time comparison between the transient model and the steady state based model

\begin{tabular}{|l|l|l|l|}
\hline & $\begin{array}{l}\text { Thermal analysis } \\
\text { time }\end{array}$ & $\begin{array}{l}\text { Mechanical analysis } \\
\text { time }\end{array}$ & $\begin{array}{l}\text { Total analysis } \\
\text { time }\end{array}$ \\
\hline Transient model & 51 hours, 24minutes & 24hours, 1minute & 75hours, 25minutes \\
\hline Steady state model & 10 minutes & 14 hours, 46minutes & 14 hours, 56minutes \\
\hline Time saving & $99.69 \%$ & $38.51 \%$ & $80.21 \%$ \\
\hline
\end{tabular}

\section{Results and discussion}

\subsection{Thermal results}


Fig. 5 presents the contour plots of temperature results of the Lagrangian thermal model and the Eulerian thermal model, respectively. Both models produced similar heating and cooling cycles.

a

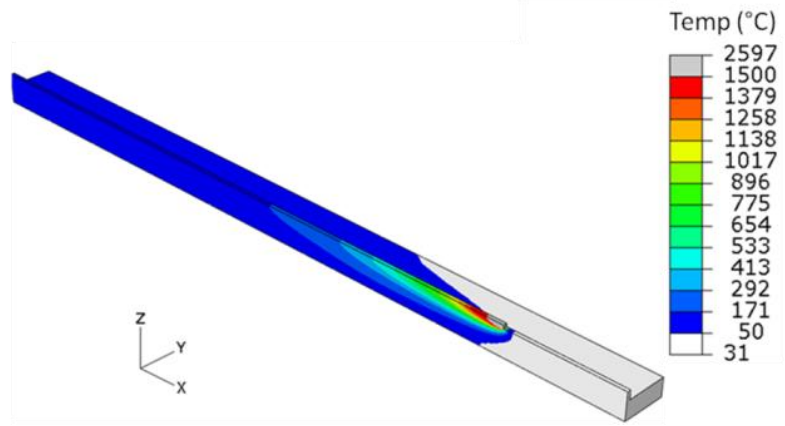

b

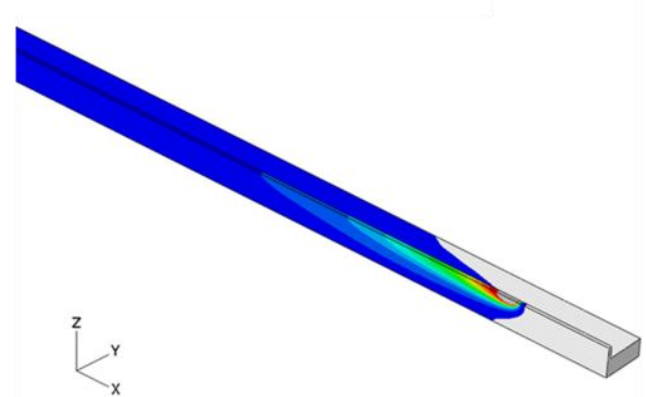

Fig. 5: Temperature distribution from: (a) transient and (b) steady-state thermal models.

The thermal models were verified by comparing the temperature history results from the four thermocouples positions on the base plate shown in Fig. 2. These results are presented in Fig. 7. As the steady-state model does not have a time related history, the thermal cycle of the testing point is obtained by plotting the temperature along lines with fixed $y$, and $\mathrm{z}$ coordinates which correspond to the thermocouple positions shown in Fig. 2. The time coordinate is found by dividing the position in the $\mathrm{x}$ direction by the travel speed.
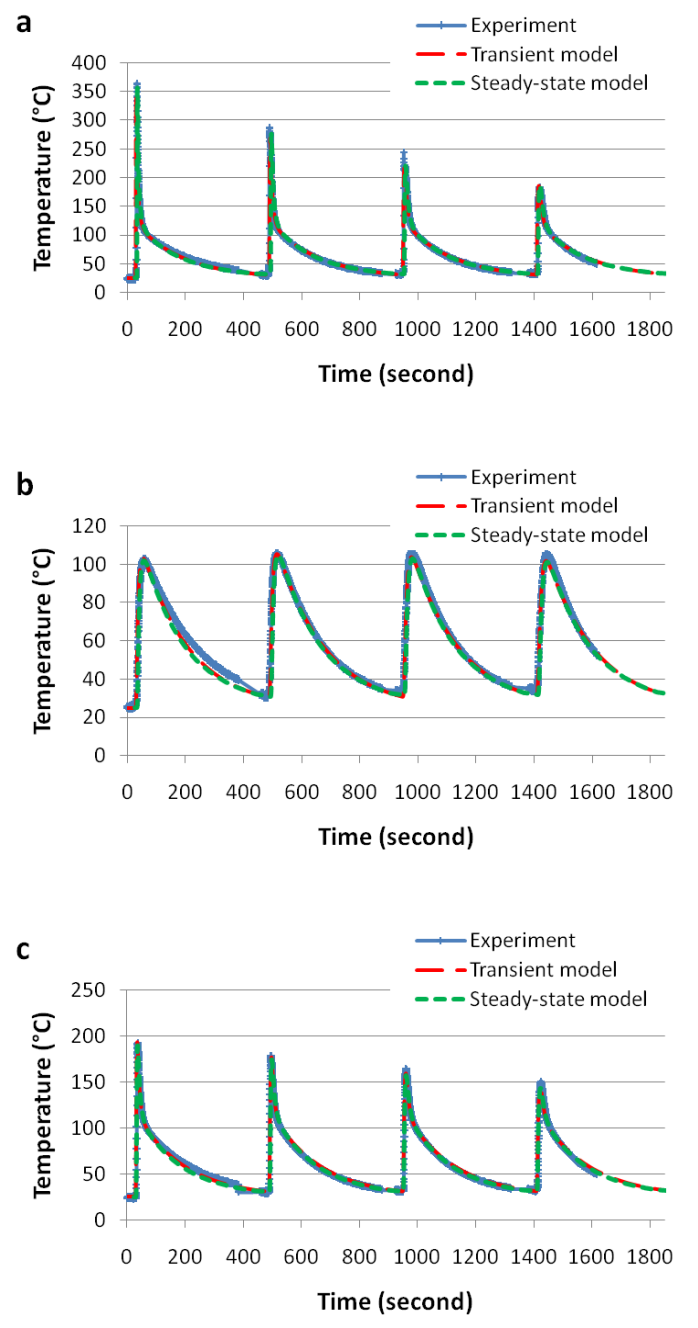


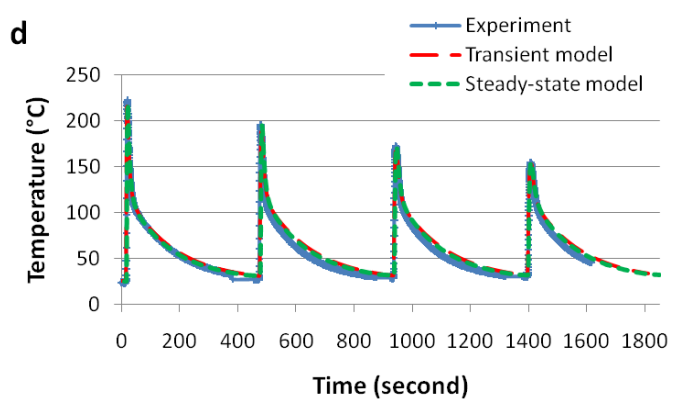

Fig. 6: Temperature verification on the measuring positions of (a) TP1 $(250,5,0),(b)$ TP2 $(250,20,0),(c)$ TP3 $(250,0,-12)$, (d) TP4 (375, 0, -8).

Both, the transient and steady-state thermal models give accurate predictions of the temperatures at the four thermocouple positions. Although the steady state model underestimates the temperature marginally because heat can be conducted via the elements behind the heat source, the effect is small because of the high travel speed.

\subsection{Mechanical results}

Fig. 7 (a) and (b) illustrate the predicted longitudinal residual stress of the four layer wall with clamping from the two model types, which indicate similar results. The heat from the welding process causes a tensile residual stress along the weld bead due to material contraction during solidification, which causes a balancing compressive residual stress in the base plate. Note that the magnitude of the compressive stress is greatest where clamping has been applied due to the restraint in this region. There is significant distortion of the component and relaxation of the stresses after the clamping was removed which is illustrated in Fig. 7(c). To better understand these stress changes, the longitudinal stress was plotted along the dotted lines in Fig. 7(b, c) in Fig. 7 (d).

Fig. 7 (d) shows that when the specimen is clamped, the highest longitudinal stress occurs in the deposited wall. The stress predicted across the deposit is very uniform with very little annealing of the earlier layers by the latter ones. To further investigate this finding, a further 4 layers were deposited (giving 8 layers in total) and the results plotted in Fig. 7 (d). There is little change in the stresses generated, further supporting this finding. Indeed Chin et al. [8] found a similar result in their model of additive layer manufacture. The stresses in the base plate adjacent to the deposited wall are also tensile, however there is a significant drop in magnitude since the material in the base is softer than the weld metal. It is only near the bottom of the base plate that the longitudinal stresses go into compression.

The significant drop in the longitudinal stress after unclamping is also shown in Fig. 7 (d). The interesting aspects of this plot occur toward the extremities. For example, the stress at the top of the deposited wall has a much lower value than at the interface due to the bending distortion of the sample. This distortion also causes the compressive stress at the bottom of the base plate to go into tension. There is a reduction in the tensile longitudinal residual stress measured in the base plate with increased layers due to the influence of bending distortion. This is reflected in the residual stress measurements shown in Fig. 7 (b) where four and eight layer walls are compared unclamped.

To have an overall check of the mechanical results, the predicted distortion along the long edge from the two mechanical models was compared with the measured distortion from the two long edges of the sample. Fig. 8 shows how the distorted shape from both models is nearly identical and agrees with the experimentally measured distortion.

a

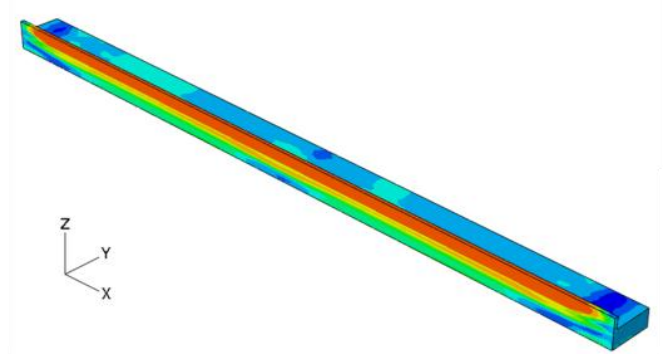

$\mathrm{b}$

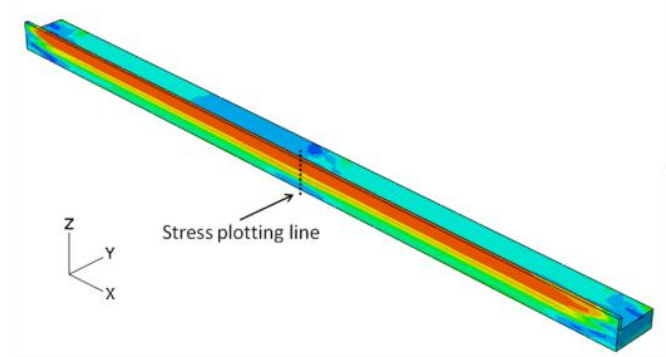



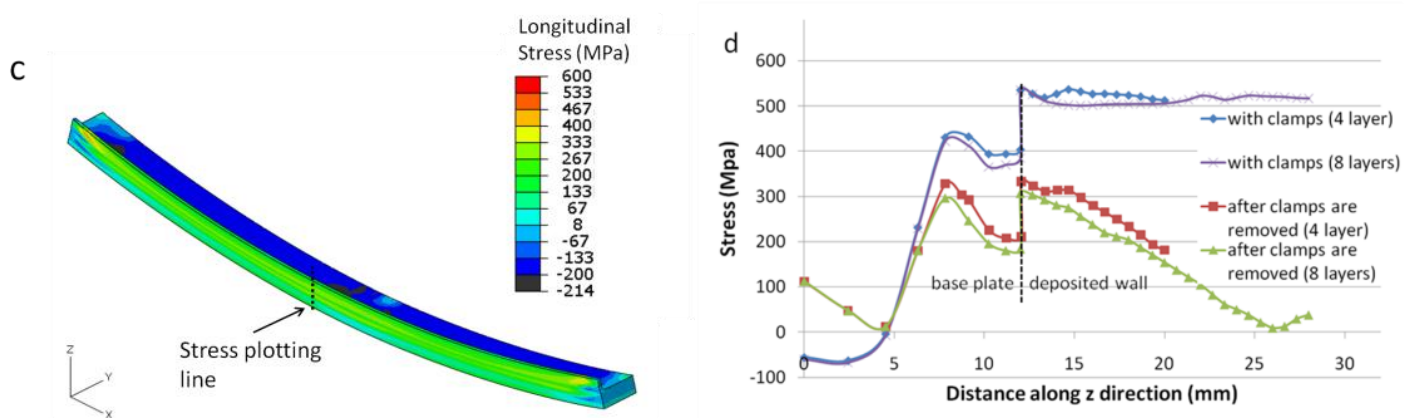

Fig. 7: Longitudinal residual stress predictions from: (a) transient thermal and mechanical models; (b) steady state thermal and mapped mechanical models; (c) steady state thermal and mapped mechanical models after clamps were removed (a scaling factor of 5 is used for the distorted shape); and (d) the stress plotted along the lines shown in of $(b)$ and $(c)$.

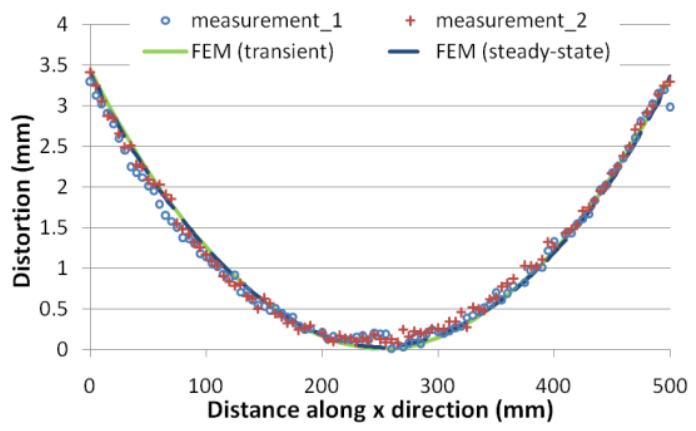

Fig. 8: Distortion verification along the longitudinal direction after the clamps were removed.

Detailed validation of the residual stress data was provided by comparing the computational results for different numbers of deposited layers with those from neutron diffraction measurements.Fig. 9 shows the comparison on the base plate. In both the modelling and experimental results the clamps have been removed, and three principle directions have been considered namely: longitudinal $(\sigma \mathrm{LD})$, transverse $(\sigma \mathrm{TD})$, and normal $(\sigma N D)$. The results are similar to those observed in long welding processes with the longitudinal stress dominating over the transverse and normal stresses [21]. The FE predictions match the experimental results well in all cases except the 1 layer wall where the model underpredicts the stresses. Moreover, there is a more significant reduction in the experimentally measured stresses between the 1 and 2 layer walls, than in the stresses predicted by the models. A possible explanation is that the model does not fully capture the microstructural changes which occur in the actual material.

A sample with 20 layer wall was made to validate the residual stress on the deposited wall. Longitudinal stress was measured without clamps in the mid-section along the normal direction of the deposited wall. Due to the long computational time the transient model need, the model based on the steady-state thermal solution was used for the prediction. The comparison demonstrated in Fig. 10 shows that the numerical prediction matches the experimental result well also on the deposited wall. The longitudinal stress goes into compression on the top layers.

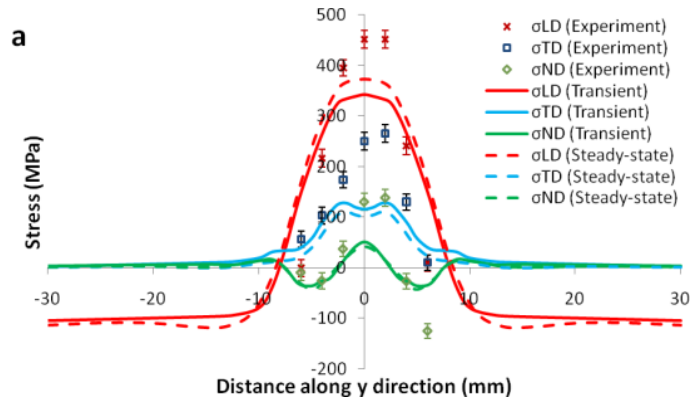




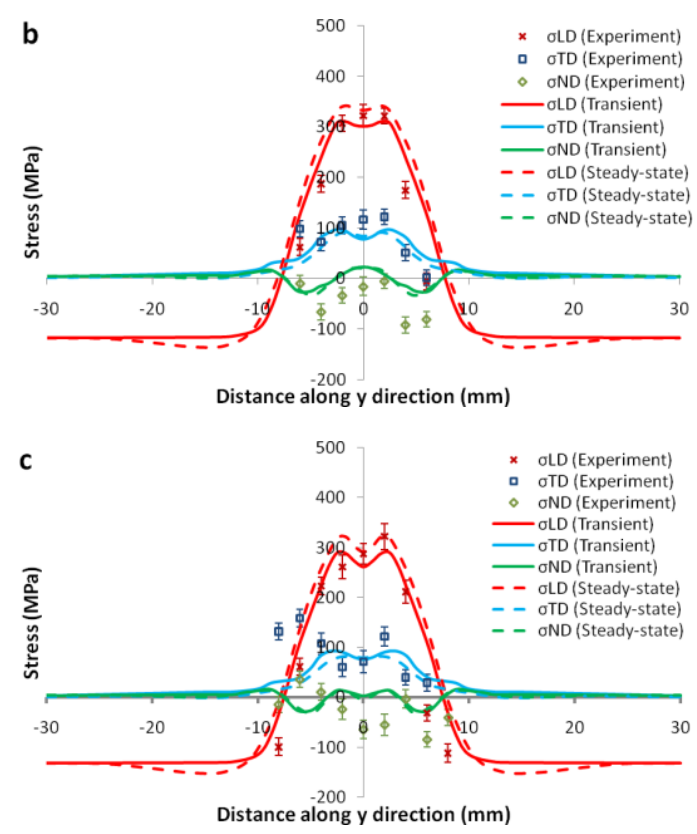

Fig. 9: Stresses in the longitudinal (LD), transverse (TD) and normal (ND) directions along a transverse line in the base-plate indicated in Fig. 2 for (a) single, (b) two, and (c) three layers.

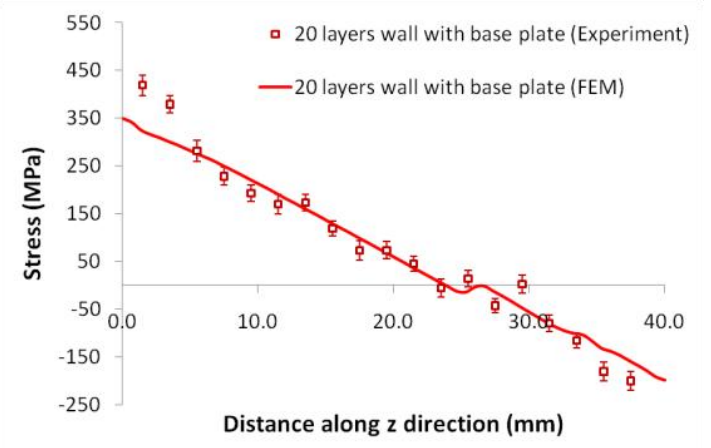

Fig. 10: Longitudinal stress along the normal direction of the mid-section in the deposited wall.

In general, unevenly distributed stresses generated during the WAALM process can cause big distortions on the parts after the clamps removed. Depending on the application, it will be necessary to provide a subsequent heat treatment to relieve these stresses and improve the mechanical properties that would be affected by tensile residual stresses. The FEM models introduced in this paper can provide accurate prediction of the stresses and distortions of the WAALM process. This will be useful for future work where the WAALM process will be optimised to minimise the residual stress and distortion.

\section{Conclusions}

In this study, the thermo-elastic-plastic FEM is employed to simulate the thermo-mechanical performance of large multi-layer wall shaped WAALM structures. Experiments are carried out to verify the temperature distribution, stress distribution and distortions. Moreover, an efficient FE method based on Eulerian steady-state thermal analysis is also discussed. According to the results in this study, the following conclusions can be drawn:

1) Both FEM models can accurately predict the heating and cooling cycles during the WAALM process. The steady-state model can also be used for dealing with the multi-layer WAALM process.

2) The stress evolution analysed using the numerical model shows that stress across the deposit is very uniform with very little influence of the preceding layers on the following layers.

3) A significant stress redistribution is observed after unclamping. The stress at the top of the deposited wall has a much lower value than at the interface due to the bending distortion of the sample. The neutron diffraction strain scanner ENGIN-X reveals a good match of the predicted stress data with the measurements. The measured distortions also support the model predictions. 
4) The new FEM approach based on the steady-state thermal model shows a significant advantage of around $80 \%$ on the computational time. This will be useful for future work where the WAALM process will be optimised to minimise the residual stress and distortion.

\section{Acknowledgement}

This research was carried out under RUAM project (131) of the IMRC (Innovative Manufacturing Research Centre) Cranfield funded by EPSRC, UK. The authors would like to thank Flemming Nielsen for his technical assistance and Dr Anna Paradowska for her support in ISIS.

\section{References}

[1] P.M. Sequeira Almeida, S. Williams: 'Innovative process model of Ti-6Al-4V additive layer manufacturing using cold metal transfer (CMT) ', Proceedings of the Twenty-First Annual International Solid Freeform Fabrication Symposium, University of Texas at Austin, Austin, TX, USA, August 9-11, 2010.

[2] D. Wood: 'Additive Layer Manufacturing at Airbus - Reality check or view into the future?' TCT, 17(2000), 23-27.

[3] R. Baker, Method of Making Decorative Articles, US Patent 1,533,300, filed Nov. 12 1920, patented 14th April 1925.

[4] F. Ribeiro, 3D Printing with Metals, Computing \& Control Engineering Journal, 9 (1998), 31-38.

[5] J.D. Spencer, P.M. Dicken, C.M. Wykes, Rapid Prototyping of Metal Parts by Three-Dimensional Welding, Proc. Inst. Mech. Eng. Part B-J. Eng. Manuf., 212(1998), 175-182.

[6] D. Clark, M.R. Bache and M.T. Whittaker, Shaped Metal Deposition of a Nickel Alloy for Aero Engine Applications, J.Mater.Process.Technol., 203(2008), 439-448.

[7] B. Baufeld, O.V.d. Biest, R. Gault, Additive Manufacturing of Ti-6Al-4V Components by Shaped Metal Deposition: Microstructure and Mechanical Properties, Mater Des, 31(2010), 106-111.

[8] 4R.K. Chin, J.L. Beuth, C.H. Amon, Successive Deposition of Metals in Solid Freeform Fabrication Processes, Part 1: Thermomechanical Models of Layers and Droplet Columns, J. Manuf. Sci. Eng., 123 (2001), 632-638.

[9] R.K. Chin, J.L. Beuth, C.H. Amon: Successive Deposition of Metals in Solid Freeform Fabrication Processes, Part 2: Thermomechanical Models of Adjacent Droplets, J. Manuf. Sci. Eng., 123 (2001), 632-638.

[10] M. Chiumenti, M. Cervera, A. Salmi, C. Agelet de Saracibar, N. Dialami, K. Matsui, Finite Element Modelling of Multi-Pass Welding and Shaped Metal Deposition Processes, Comput.Methods Appl.Mech.Eng., 199(2010), 2343-2359.

[11] H. Fawad, M. P.Mughal, R.A., Mufti, Effect of constraint on residual stresses and deformations in weld based rapid prototyping, Proc. 3rd International Conf. on Advanced Research in Virtual and Rapid Prototyping: Virtual and Rapid Manufacturing, Advanced Research Virtual and Rapid Prototyping, Leiria; 24 - 29 September 2007, 629-634

[12] M. P. Mughal, R. Mufti, H. Fawad, the Mechanical Effects of Deposition Patterns in Welding-Based Layered Manufacturing, Proc. Inst. Mech. Eng. Part B-J. Eng. Manuf., 221(2007), 1499-1509.

[13] J.Y. Shanghvi, P. Michaleris, Thermo-Elasto-Plastic Finite Element Analysis of Quasi-State Processes in Eulerian Reference Frames, Int. J. Numer. Mech. Engng, 53(2002), 1533-1556.

[14] X.L. Qin, P. Michaleris, Elasto-Visco-Plastic Analysis of Welding Residual Stress, Sci. Technol. Weld. Joining, 14(2009), 606-615.

[15] S. Wang, J. Goldak, J. Zhou, S. Tchernov, D. Downey, Simulation on the Thermal Cycle of a Welding Process by space-time convection-diffusion Finite Element Analysis, Int. J. Therm. Sci., 48 (2009), 936-947.

[16] L. Zhang, P. Michaleris, Investigation of Lagrangian and Eulerian Finite Element Methods for Modeling the Laser Forming Process, Finite Elements Anal.Des., 40(2004), 383-405.

[17] M. Gu, J. Goldak, Steady-State Formulation for Stress and Distortion of Welds, J Eng Ind Trans ASME, 116 (1994), 467-474.

[18] P. Colegrove, C. Ikeagu, A. Thistlethwaite, S. Williams, T. Nagy, W. Suder, A. Steuwer, T. Pirling, Welding Process Impact on Residual Stress and Distortion, Sci. Technol. Weld. Joining, 14 (2009), 717-725.

[19] ABAQUS Inc: available at: http://www.abaqus.com

[20] J. Goldak, A. Chakravarti, M. Bibby, New Finite Element Model for Welding Heat Sources, Metall. Trans. B, 15B (1984), 299-305. 
[21] X.Y. Shan, M.J. Tan, N.P. O’Dowd, Developing a Realistic FE Analysis Method for the Welding of a NET Single-Bead-on-Plate Test Specimen, J.Mater.Process.Technol. 192-193 (2007), 497-503.

[22] D. Deng, FEM prediction of welding residual stress and distortion in carbon steel considering phase transformation effects, Materials and Design, 30(2009), 359-366.

[23] C.G. Pickin, K. Young, Evaluation of cold metal transfer (CMT) process for welding aluminium alloy, Sci. Technol. Weld. Joining, 11(2006), 583-585.

[24] J. A. Dann, M.R. Daymond, L. Edwards, J.A. James, J. Santisteban, A comparison between Engin and Engin-X, a new diffractometer optimized for stress measurement, Physica B, 350(2004), 511-514.

[25] J.R. Santisteban, M.R. Daymond, J.A. James, L. Edwards, ENGIN-X: A third-generation neutron strain scanner, J. Appl. Crystallogr, 39(2006), 812-825.

[26] G. S. Pawley, Unit-cell refinement from powder diffraction scans, J. Appl. Crystallogr,14(1981), 357-361. 